\title{
Student difficulties with complex numbers
}

\author{
Emily M. Smith, ${ }^{1}$ Justyna P. Zwolak, ${ }^{2}$ and Corinne A. Manogue ${ }^{1}$ \\ ${ }^{1}$ Department of Physics, Oregon State University, Corvallis, OR 97331 \\ ${ }^{2}$ STEM Transformation Institute, Florida International University, Miami, FL 33199
}

\begin{abstract}
Complex numbers and functions are used in multiple subfields in undergraduate physics. We use pretests, quizzes, and exams administered throughout the junior year to identify middle-division students' difficulties with complex number fluency. These difficulties are classified into three categories: performing calculations, switching between forms, and appropriately selecting forms to simplify calculations. Our exploration suggests that students in middle-division physics courses have varying levels of fluency with complex number manipulations. Some of these difficulties persist over time.
\end{abstract}

PACS numbers: 01.40.Fk, 01.40.gb

\section{INTRODUCTION}

At Oregon State University, as at many institutions, physics majors are introduced to, and use, complex numbers and functions in several courses. Faculty suspect, from exam and homework performance in quantum mechanics courses, that student difficulties with elementary complex number and function manipulations cause persistent problems throughout the undergraduate curriculum. As a first step to remedy this situation, we conducted a baseline study to identify the experiences we are giving students with complex numbers and find out how effective those experiences are. In this paper, we describe some of the difficulties that undergraduate physics students encounter throughout the junior year with complex number fluency including calculations and changing between, and appropriately using, different forms.

Previous empirical studies explore secondary students' [1], undergraduate students' [2-5], prospective and inservice secondary teachers' [6-8], and experts' [9] algebraic and geometric understanding of complex numbers in mathematics and engineering contexts. Some of these studies cover calculational aspects of complex number fluency we discuss including complex algebra [2] and changing between [8], and appropriately selecting [4], forms of complex numbers. While one study included a physics expert [9], we were unable to identify studies which focus on undergraduate physics students' understanding of complex numbers.

In the Paradigms in Physics program at OSU, the content of the courses was extensively reordered as a result of a reform instituted in 1997. While, in traditional curricula, courses focus on subfields of physics, at OSU most of the junior-level courses - called Paradigms - revolve around concepts underlying those subfields (e.g. energy, symmetry, eigenstates) [10]. Table I shows a schedule for the junior year at OSU with courses that use complex numbers and functions shown in bold.

In Week 7, students are first briefly introduced to complex numbers in class and are given relevant homework problems. Students are expected to gain familiarity with complex number and function manipulations, including operations such as multiplication, division, addition, sub-
TABLE I: Schedule of the relevant assessments and junior year courses in 2015 (for full schedule see Ref. [10]). Courses, during which students are exposed to complex numbers or functions are in bold, and those with formal review are marked with an asterisk $(*)$.

\begin{tabular}{|c|c|c|c|}
\hline Week & Assessment & \multicolumn{2}{|c|}{ Course (Junior Year) } \\
\hline \multirow{3}{*}{ 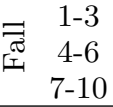 } & & \multicolumn{2}{|c|}{ Symmetries \& Idealizations } \\
\hline & & \multicolumn{2}{|c|}{ Static Vector Fields } \\
\hline & & \multicolumn{2}{|c|}{ Oscillations* } \\
\hline \multirow{4}{*}{ 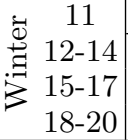 } & $\mathrm{P} 1, \mathrm{Q} 1$ & \multicolumn{2}{|c|}{ Preface* } \\
\hline & & \multirow{2}{*}{\multicolumn{2}{|c|}{$\begin{array}{c}\text { Quantum Measurements \& Spin } \\
\text { One-Dimensional Waves }\end{array}$}} \\
\hline & & & \\
\hline & & \multicolumn{2}{|c|}{ Periodic Systems } \\
\hline \multirow{5}{*}{ 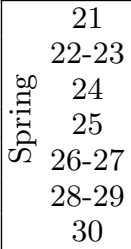 } & $\mathrm{P} 2, \mathrm{Q} 2$ & \multirow{5}{*}{ Math Methods* } & Reference Frames \\
\hline & & & \multirow{3}{*}{ Energy \& Entropy } \\
\hline & Midterm & & \\
\hline & & & \\
\hline & Final & & Central Forces \\
\hline
\end{tabular}

traction, complex conjugate, and norm, in rectangular, polar, and exponential forms. Homework problems include switching forms and plotting on Argand diagrams. For each homework problem more than $70 \%$ of students $(\mathrm{N}=105)$ provided correct solutions. Furthermore, students use complex functions during Weeks 8-10 to solve differential equations for harmonic motion. In Math Methods, simultaneous with Paradigms in Weeks 21-30, there is a review of complex number forms, representations, and operations prior to more advanced complex analysis topics including complex-valued power series and analyticity. Supported by the results from homework in Week 7, instructors have assumed students are fluent with complex number manipulations prior to Weeks 11-30, which are the subject of this study.

\section{METHODOLOGY}

We describe the curricular experiences that students have with complex algebra in Weeks 11-30 and describe six in-class assessments containing open-ended prompts 
to track students' increasing ability to do simple complexnumber calculations. Timing of these assessments is shown in Table I, During these weeks, two unannounced and ungraded pretests (P1, P2), two announced quizzes (Q1, Q2), and explicit questions on two exams (Midterm, Final) were administered to gauge students' abilities to perform calculations involving complex numbers.

Students enrolled in the Paradigms are primarily physics majors but occasionally include physics minors, related majors, and graduate students. In a typical year, 30-37 students are enrolled in each Paradigm, and most majors complete the sequence in the intended order. For Weeks 11-20, we use data from all students who completed each task. From Weeks 21-30, we excluded data from graduate students from other fields because of differences in their background. Data has been collected sporadically over the past four academic years; not all tasks have been administered each year which accounts for differences in the number of student responses for each assessment. Since we did not notice significant performance differences from year to year, all available responses are included in this baseline study.

Each assessment was analyzed to find the percentage of students answering correctly. Incorrect answers were then sorted by type of error, as a first step in a grounded theory approach [11]. Tables II and III show results from $\mathrm{P} 1, \mathrm{Q} 1$, and $\mathrm{P} 2$ in which students were asked to find the square and the norm squared of a complex number. Tables IV and V show results from P2, Q2, Midterm, and Final (administered only in Spring 2015) in which students are asked explicitly to change from one form of a complex number to another or to calculate the natural logarithm where it is necessary for students to correctly select the form.

The forms for the complex number, $z$, which we explore are the rectangular form given by $x+i y$, the polar form given by $r \cos \theta+i r \sin \theta$, and the exponential form given by $r e^{i \theta}$ where $x, y, r$, and $\theta$ are real numbers.

\section{CALCULATION DIFFICULTIES}

During Week 11, a 10 minute in-class review of complex numbers includes relating forms, geometric relationships, and common operations. A short kinesthetic activity where students represent complex numbers and operations using their arms is incorporated [12]. P1 and Q1, given on the days immediately before and after the review, respectively, were used to determine students' ability to perform calculations of the square and norm squared, given symbolically, in rectangular and exponential forms. For example, Q1 administered in 2015 included questions: "For $z_{1}=-3+7 i$, find $z_{1}^{2}$ and $\left|z_{1}\right|^{2}$ " and "For $z_{2}=3 e^{2 i}$, find $z_{2}^{2}$ and $\left|z_{2}\right|^{2}$ ".

The responses, including specific errors, which students gave when determining the square of a complex number are summarized in Table II. A common difficulty was failure to correctly use the distributive property on a
TABLE II: Comparison of difficulties with squaring a complex number in rectangular (P1: $\mathrm{N}=86, \mathrm{Q} 1: \mathrm{N}=115)$ and exponential (P1: $\mathrm{N}=54, \mathrm{Q} 1: \mathrm{N}=83, \mathrm{P} 2: \mathrm{N}=23$ ) forms. Other includes responses unique to one or two individuals.

\begin{tabular}{c|c|c||c|c|c}
\hline$z^{2}$ & \multicolumn{2}{|c||}{ Rectangular Form } & \multicolumn{3}{c}{ Exponential Form } \\
& P1 [\%] & Q1 [\%] & P1 [\%] & Q1 [\%] & P2 [\%] \\
\hline Correct & 58.1 & 80.0 & 57.4 & 73.5 & 69.6 \\
No Response & 9.3 & 0.9 & 31.5 & 7.2 & 4.3 \\
Distributive & 10.5 & 10.4 & - & - & - \\
$i^{2}=1$ & 10.5 & 4.4 & - & - & - \\
$|z|^{2}$ & 7.0 & 0.9 & 0 & 0 & 0 \\
$r e^{2 i \theta}$ & - & - & 7.4 & 7.2 & 4.3 \\
Other & 4.7 & 3.5 & 3.7 & 12.0 & 21.7 \\
\hline
\end{tabular}

complex number in rectangular form. This includes both minor arithmetic errors with multiplication and neglecting the sign of $i^{2}$ (listed separately in Table II as $i^{2}=1$ ). We have included these as distinct categories because we are unsure whether students do not recall the sign of $i^{2}$ or whether this is a simple arithmetic error. Other authors found distributivity errors in similar problems with undergraduate engineering students, but further foundational errors such as stating $i^{2}=0$ or ignoring $i$ were not observed with the Paradigms students [2]. Additionally, in rectangular form, some students $(7.0 \%, \mathrm{~N}=86)$ found the norm squared rather than the square on $\mathrm{P} 1$. This could be due to instructional language which may, at times, fail to distinguish between the square and norm squared by referring to both operations as the "square".

For the exponential form nearly a third of students $(31.5 \%, \mathrm{~N}=54)$ did not provide a response on $\mathrm{P} 1$. This could be indicative of calculational difficulties with exponentials. Additionally, responses with only the square of the phase portion (given in Table II as $r e^{2 i \theta}$ ) could be either arithmetic errors or could be foundational.

P1 and Q1 were also used to determine students' ability to calculate the norm squared; difficulties are summarized in Table III. Many students do not provide any response for the norm squared in rectangular $(34.9 \%$, $\mathrm{N}=86)$ and exponential $(61.1 \%, \mathrm{~N}=54)$ forms. Likely this is indicative of not knowing what operation the symbols represent. Of those who respond, simple arithmetic errors do occur, but the most prevalent incorrect responses are operations which are not the norm squared. Calculations of $\left(|z|^{2}\right)^{2}$ and $|z|$ require knowledge of the mathematical process of determining the norm squared, however, students responding in these ways possibly do not correctly distinguish the norm and norm squared. Students responding with solutions which are of the form, $\left(z^{2}\right)^{*}$, likely recognize there is a difference between the square and norm squared but fail to recall the proper operation (though they perhaps know there is a step involving the complex conjugate). Though few students responded with either $x^{2}-y^{2}$ or $x^{2}$ for rectangular form in $\mathrm{P} 1$, these responses may show that students recognize that the norm squared is a real quantity but obtained this incorrectly by either taking the real part of the square or 
TABLE III: Comparisons of difficulties for norm squared in rectangular (P1: $\mathrm{N}=86, \mathrm{Q} 1: \mathrm{N}=115, \mathrm{P} 2: \mathrm{N}=23$ ) and exponential (P1: $N=54, \mathrm{Q} 1: \mathrm{N}=83, \mathrm{P} 2: \mathrm{N}=23$ ) forms. Other includes responses unique to one or two individuals.

\begin{tabular}{c|c|c|c||c|c|c}
\hline$|z|^{2}$ & \multicolumn{3}{|c|}{ Rectangular Form } & \multicolumn{3}{c}{ Exponential Form } \\
& P1[\%] & Q1[\%] & P2[\%] & P1[\%] & Q1[\%] & P2[\%] \\
\hline Correct & 37.2 & 79.1 & 78.3 & 20.4 & 66.3 & 87.0 \\
No Response & 34.9 & 2.6 & 4.3 & 61.1 & 15.7 & 0 \\
Arithmetic & 1.2 & 8.7 & 4.3 & 0 & 1.2 & 0 \\
$\left(|z|^{2}\right)^{2}$ & 3.5 & 1.7 & 13.0 & 1.9 & 3.6 & 13.0 \\
$|z|$ & 2.3 & 2.6 & 0 & 3.7 & 3.6 & 0 \\
$z^{2}$ & 1.2 & 0 & 0 & 7.4 & 1.2 & 0 \\
$\left(z^{2}\right)^{*}$ & 5.8 & 0.9 & 0 & 0 & 1.2 & 0 \\
$x^{2}-y^{2}$ & 3.5 & 0.9 & 0 & - & - & - \\
$x^{2}$ & 3.5 & 0 & 0 & - & - & - \\
Other & 7.0 & 3.5 & 0 & 5.6 & 7.2 & 0 \\
\hline
\end{tabular}

squaring the real part.

Student improvement from P1 to Q1 demonstrates that many, but not nearly all, students quickly learn to determine and distinguish between the square and norm squared for both rectangular and exponential forms when these calculations are addressed in-class. However, the announced and graded nature of Q1 may also be a factor. It is likely that many students at the beginning of the junior year are not familiar with these calculations involving complex numbers, particularly the norm squared and using exponential form. Given the review in Week 7 and the use of complex functions in the context of harmonic motion from Weeks 7-10, we would expect nearly all students to be able to perform these simple calculations prior to Week 11. While the short review during Week 11 addressed calculation difficulties with the square and norm squared in both forms, these are skills that students should be retaining from Week 7 .

P2 was intended to determine the retention of students' abilities to perform these calculations 10 academic weeks following the in-class review and therefore includes tasks equivalent to P1. During these 10 weeks there is no formal instruction on complex numbers, however, students extensively use complex numbers and functions in the context of spin- $1 / 2$ and spin- 1 systems, one-dimensional waves, and coupled harmonic oscillators in both classical and quantum mechanics. This instruction includes kinesthetic activities, which build on the Week 11 complex number review, to represent overall and relative phases for time-independent and time-dependent quantum systems [12].

After approximately 15 homework problems from Weeks 11-20 which require calculation of the norm squared in physics contexts, $\mathrm{P} 2$ shows both retention and improvement in student performance. Many of the difficulties with the square in rectangular form on P2 are different from those in Table II because many students $(39.1 \%, \mathrm{~N}=23)$ switched to exponential form then calculated the square. By Week 21, most students recognize exponential form as preferred for performing certain calculations, however, P2 did not appropriately gauge reten-
TABLE IV: Student responses by difficulties changing from exponential to rectangular ( $\mathrm{P} 2$ : $\mathrm{N}=23, \mathrm{Q} 2: \mathrm{N}=23$ ) and rectangular to exponential (P2: $\mathrm{N}=23$, Final: $\mathrm{N}=21$ ) forms.

\begin{tabular}{c|c|c||c|c}
\hline & \multicolumn{2}{|c||}{ Exp. $\rightarrow$ Rect. } & \multicolumn{2}{c}{ Rect. $\rightarrow$ Exp. } \\
& P2 [\%] & Q2 [\%] & P2 [\%] & Final [\%] \\
\hline Correct & 47.8 & 60.9 & 26.1 & 61.9 \\
No Response & 4.3 & 0 & 13.0 & 9.5 \\
Not Simplified & 13.0 & 4.3 & 21.7 & 0 \\
Trigonometry & 17.4 & 21.7 & 13.0 & 9.5 \\
$\theta=\tan ^{-1}\left(\frac{x}{y}\right)$ & - & - & 8.7 & 0 \\
Other & 17.4 & 13.0 & 17.4 & 19.0 \\
\hline
\end{tabular}

tion of squaring a complex number in rectangular form.

\section{CHANGING \& SELECTING FORMS}

At the beginning of Math Methods in Week 21 and immediately following P2, 15 minutes of review included forms, representations, and operations with an emphasis on switching forms and selecting forms for particular operations (e.g. rectangular form for addition, exponential form for multiplication). The following class emphasized selecting forms based on the mathematical context and began to introduce complex functions in both rectangular and exponential forms. The final class during Week 21 included a lecture on multiple-valued complex functions with examples similar to problems given on the Midterm and Final.

At the beginning of Week 21, as assessed by P2, many students had difficulties changing from exponential (given as $3 e^{-i \pi / 4}$ ) to rectangular form. As a follow-up assessment, at the beginning of the third class in the same week, Q2 included a problem to express $3+3 e^{-3 i \pi / 4}$ in rectangular form; we consider the step where students change $3 e^{-3 i \pi / 4}$ to rectangular form. While more students $(60.9 \%)$ correctly changed forms on Q2 than P2 $(47.8 \%)$, possibly due to the in-class instruction, there were many student difficulties evaluating trigonometric quantities - failing to simplify from polar form (listed separately as Not Simplified), sign issues, and incorrect geometry - on both P2 (30.4\%) and Q2 (26.0\%) as shown in Table IV $(\mathrm{N}=23)$.

Problems involving changing from rectangular to exponential form included a prompt on $\mathrm{P} 2$, to express $\sqrt{3}-i$ in exponential form, and a question on the Final, 10 weeks later, to express $\ln \left[(i-1)^{10}\right]$ in rectangular form. For the Final, we analyzed only the responses from students who attempted to change $i-1$ to exponential form. While student improvement in navigating from rectangular to exponential forms is considerable from P2 $(26.1 \%, \mathrm{~N}=23)$ to the Final $(61.9 \%, \mathrm{~N}=21)$, student difficulties switching from rectangular to exponential form, summarized in Table IV, seem to primarily originate from relying on equations (e.g. $\theta=\tan ^{-1}\left(\frac{y}{x}\right)$ ) without considering the geometry relating the forms. On the Final, few students $(9.5 \%, \mathrm{~N}=21)$ made trigonometry related errors, how- 
TABLE V: Midterm and Final $(\mathrm{N}=25)$ correct and incorrect responses by primary form used within the logarithm.

\begin{tabular}{c|cc||cc}
\hline \multirow{2}{*}{$\ln z$} & \multicolumn{2}{|c||}{ Midterm } & \multicolumn{2}{c}{ Final } \\
& Correct[\%] & Incorrect[\%] & Correct[\%] & Incorrect[\%] \\
\hline Exp. & 28.0 & 24.0 & 32.0 & 48.0 \\
Rect. & 0 & 48.0 & 0 & 8.0 \\
Other & - & - & 4.0 & 8.0 \\
\hline
\end{tabular}

ever, the geometry associated with $i-1$ may be simpler for students than $\sqrt{3}-i$. This may be similar to other studies which showed in-service secondary mathematics teachers also exhibited a lack of flexibility between forms of complex numbers despite extensive instruction [8].

In order to evaluate the form students chose within a particular mathematical context, both the Midterm and Final included a problem with a natural logarithm; students responses are summarized by primary form used within the natural logarithm in Table V. On the Midterm, half of students $(52 \%, \mathrm{~N}=25)$ primarily used the rectangular form to try to determine the real and imaginary parts of $\ln \left(\frac{1}{z}\right)$ - demonstrating difficulty in selecting the appropriate form and preference for rectangular form which is similar to another study involving undergraduates enrolled in a complex analysis course [4].

During Week 21, the instructor gave an in-class example of $\ln z$ using exponential form, and homework problems emphasized selecting an appropriate form given the mathematical context. Combined with extensive use of complex numbers and functions in preceding Paradigms, it was unexpected that students would encounter difficulties related to selecting a form. By the Final most students $(80 \%, \mathrm{~N}=25)$ recognized the exponential form as the correct form to use. Despite not reaching the correct solution, students have improved in their selection of an appropriate form from the context of the mathematics; this may be due to the similar Midterm problem and further experience with complex functions in ordinary and partial differential equations during Weeks 25-30.

\section{CONCLUSIONS}

Complex algebra may be difficult for students for many reasons: their lack of prior experience, our failure to provide students with an extended introduction, and existing difficulties with unit circle trigonometry essential to switching forms of complex numbers. Despite the apparent improvement from Weeks 21-30, we are still disappointed with the number of students encountering difficulties this late in the junior year especially with the several (brief) treatments of complex numbers throughout the curriculum. This dynamic may be common to many upper-division curricula, so the results of this study may be generalizable beyond the specific context of the Paradigms curriculum. Students who cannot master simple manipulations, such as distinguishing between and calculating the square and norm squared, switching forms, and selecting an appropriate form, will not be successful in completing upper-division problems, especially in quantum mechanics, where complex numbers and functions are pervasive.

Because OSU students experience these difficulties, we plan to develop a thorough introduction for the incoming juniors this Fall which emphasizes geometry relating the forms, similarities with two-dimensional vectors, and practice with calculations. We intend to study whether implementing an early intervention in the junior year enables students to demonstrate greater fluency with complex number algebra as they progress through the middledivision and into the upper-division physics courses.

\section{ACKNOWLEDGMENTS}

Partially supported by NSF grant DUE 1323800.
[1] A. Panaoura, I. Elia, A. Gagatsis, and G.P. Giatilis, IJMEST 37(6), 681 (2006).

[2] A.W. Ahmad and M. Shahrill, IJSR 3(8), 273 (2014).

[3] M.C. Nordlander and E. Nordlander, IJMEST 43(5), 627 (2012).

[4] P. Danenhower, Ph.D. thesis, Simon Fraser University, 2000.

[5] H. Soto-Johnson and J. Troup, JMB 36, 109 (2014).

[6] M.E. Conner, C. Rasmussen, M. Zandieh, and M. Smith, in Proceedings of the Conference on Research in Undergraduate Mathematics Education (Mathematical Association of America, San Diego, 2007).

[7] R. Nemirovsky, C. Rasmussen, G. Sweeney, and M. Wawro, J. Learn. Sci. 21(2), 287 (2012).
[8] G. Karakok, H. Soto-Johnson, and S. Dyben, JMTE p. 1-25 (2014).

[9] H. Soto-Johnson, M. Oehrtman, and S. Rozner, in Proceedings of the Conference on Research in Undergraduate Mathematics Education edited by S. Brown et. al (Mathematical Association of America, Portland, 2012), Vol. 1, p. 433.

[10] physics.oregonstate.edu/portfolioswiki/courses:start.

[11] D.F. Lancy, Qualitative Research in Education (Longman, New York, 1993) p. 10.

[12] physics.oregonstate.edu/portfolioswiki/whitepapers: sequences:complex. 